\title{
Guidelines on calculation of the concrete thermal treatment modes
}

\author{
Sergey Novikov*, Boris Zhadanovsky and Sergey Sinenko \\ Moscow State University of Civil Engineering, Department of Construction Technology and \\ Organization, Yaroslavskoe chaussee 26, Moscow, Russian Federation
}

\begin{abstract}
The article provides guidelines on calculation of the cast-inplace reinforced concrete thermal treatment modes: the problem of thermal treatment mode by setting the mathematical temperature field model in the hardening concrete has been solved; model equation which allows calculating the temperature field in the hardening concrete structure using computer software has been solved. These data allow to achieve the high quality of concrete at minimum heat treatment duration and the maximum reduction of energy costs, while minimizing experimental calculations.
\end{abstract}

\section{The energy efficiency problem of economy class residential buildings}

One of the most pressing social problems in the world is the problem of housing for citizens, towards whom the state has obligations in accordance with applicable law. Currently, the most efficient way to solve this problem directly is a mechanism of provision of housing at the expense of state funds. Successful completion of such programs is also achieved due to the formation of an affordable economy class housing market that meets the requirements of energy efficiency and environmental friendliness. In turn, the energy efficiency criterion is achieved by rational choice of technology and organization of construction operations $[1,2]$.

\subsection{Energy efficient concrete temperature conditions}

Currently, the use of technology and organization of construction by cast-in-place method, which has a significantly accelerated level of adoption, has been increasingly recognized in the construction industry. An important criterion for energy efficiency of cast reinforced concrete technology utilization is the rational temperature condition of concrete hardening [3]. It is necessary to have a common method of the temperature field calculation in the hardening concrete structure for a satisfactory resolution of this problem. The implementation of this calculation method by using computer modelling is currently possible and relevant due to the widespread use of computer technology $[4,5]$.

\footnotetext{
*Corresponding author: $\underline{622040 @ \text { mail.ru }}$
} 


\subsection{The task of searching for the concrete structures thermal treatment modes}

Under the influence of non-homogeneous and non-stationary temperature field on the concrete hardening the thermo-stress state arises, which can lead to the ultimate structural strength reduction [5]. Therefore, it is necessary to know the temperature fields dynamics in concrete structures for different heat treatment methods. Choosing the most efficient mode of concrete structures thermal treatment, it is possible to provide high quality of concrete at the minimum duration of thermal treatment and maximum reduction of energy costs [6]. During the concrete structures thermal treatment the temperature field in the structure can be controlled by changing the initial concrete temperature, heating elements power and heat-exchange conditions on the structure surface. There appears the problem of searching of such thermal treatment mode, under which the temperature field has the required characteristics. These characteristics include temperature, rate of its rise and temperature gradient [7].

\subsection{Basic steps of the thermal treatment problem solving}

The first step of the thermal treatment problem solving is the creation of accurate mathematical model of temperature field in the hardening concrete. The second step should be devoted to the numerical solution of model equations which allow calculating the temperature field in the hardening concrete structure using the computer software. With this method, it is possible to investigate the temperature field dynamics under different thermal treatment modes and develop the most rational modes, without recourse to a large series of scientific experiments by using the software [8].

\section{The method of temperature field calculation of concrete}

The proposed new method of temperature field calculation can be applied in various ways of concrete thermal treatment, during choosing the capacity and heat insulation of the designed heating formwork, as well as for the calculation of the thermal conditions of buildings and constructions.

\subsection{Mathematical model of the temperature field}

The numerical solution of model equations defined by locally one-dimensional scheme (LOS) of the total approximation method is adopted with the above mathematical model of the temperature field in the hardening concrete structure of arbitrary shape with different heat exchange conditions on the surface.

\subsubsection{Warm water equation}

Heat transfer in the concrete in the absence of intense sources (drains) of water and steam is mainly determined by the thermal conductivity [9]. The thermal conductivity equation for three-dimensional area $\mathrm{G}$ is written in formulas 1-2 as:

$$
C \frac{\partial u}{\partial t}=L u+f, L u=\sum_{p=1}^{3} L_{p} u
$$




$$
L_{p} u=\frac{\partial}{\partial x_{p}}\left(\lambda_{p} \frac{\partial u}{\partial x_{p}}\right), \vec{x}=\left(x_{1}, x_{2}, x_{3}\right) \in G
$$

The formulas (1-2) content the next variables:

- $C$ - specific heat per unit volume, $\mathrm{J} / \mathrm{m}^{3} \cdot{ }^{\circ} \mathrm{C}$;

$-\lambda_{P}-$ thermal conductivity coefficient, $\mathrm{W}\left(\mathrm{m} \cdot{ }^{\circ} \mathrm{C}\right)$;

- $x_{P}(\mathrm{P}=1 ; 2 ; 3)$ - Cartesian coordinates, $\mathrm{m}$;

- $t$ - time, $\mathrm{s}$;

- $u$ - temperature, ${ }^{\circ} \mathrm{C}$;

- $f$ - internal heat sources intensity, $\mathrm{W} / \mathrm{m}^{3}$.

\subsubsection{Domain task}

Area G must include not only the fresh concrete the temperature field of which is under consideration, but also solid parts in contact with it (for example, previously poured concrete, foundation soil, etc.). It is necessary to establish conditions on the part of boundaries $\mathrm{J}$ of $\mathrm{G}$ area in a solid:

$$
\left.u\right|_{J}=\text { const } \text { or }\left.\frac{d u}{d \vec{n}}\right|_{J}=0
$$

Values $C, \lambda_{P}$ and $f$ in Equation (1) are not the same in different zones of $\mathrm{G}$ area, i.e. they depend on coordinates due to the non-heterogeneity.

\subsubsection{Reinforcement influence}

Steel reinforcement in concrete typically occupies a small relative volume, therefore its influence on values $C$ and $f$ is not significant. However, since the steel thermal conductivity $\left(\lambda_{P}\right)$ is greater than $\lambda_{P}$ of concrete, then the heat flows spreading over the reinforcement bars have to be taken into account. Thus, $\lambda_{P}$ also depends on the location and direction of reinforcement (anisotropy of medium).

\subsubsection{Internal heat sources}

Intensity of internal heat sources consists of the energy inflow rate from outside (electric curing etc.) and intensity of concrete heat release [5]. The first term is the control action and is given as coordinate and time function. Concrete heat release intensity depends on the temperature and concrete state [6]. It should be noted that the thermal conductivity of hardening concrete also depends on temperature and its state. Currently, this issue needs shall be experimentally studied.

\subsection{Concrete heat release kinetics equation}

The heat release intensity of hardening concrete at any given time is determined by the temperature and concrete state at this point [9].

\subsubsection{Concrete state parameter}

Fundamentally it is possible to determine the state of hardening concrete completely by a 
certain set of physical parameters, which may vary in the hardening process. If the concrete has no destructive changes its state is determined by the concrete mix composition and a certain parameter monotonically changing in the concrete hardening process. Thus, it may be assumed that the physical parameters are uniquely expressed in terms of their initial values and this generic parameter. As such it is convenient to assume the concrete specific heat release $Q\left(\mathrm{~J} / \mathrm{m}^{3}\right)$ or relative heat release where $Q_{\max }$ is the maximum concrete specific heat release $\left(\mathrm{J} / \mathrm{m}^{3}\right)$.

\subsubsection{General kinetics equation of the concrete heat release}

According to the above, the concrete heat release intensity is a temperature function and relative heat release or:

$$
\begin{array}{r}
\frac{d Q}{d t}=Q_{\max } E(u, \omega) \\
\frac{d \omega}{d t}=E(u, \omega)
\end{array}
$$

The formulas $(4,5)$ content $E(u, \omega)$ - heat release function coefficients determined by the concrete mix composition.

This equation is the concrete state equation and, simultaneously, heat release kinetics equation, because the relative concrete heat release $(\omega)$ is selected as the state parameter. The heat release function can be presented as the product of temperature function $\varphi$ and state function $\psi$ :

$$
E(u, \omega)=\varphi(u) \psi(\omega)
$$

\subsection{Calculation method of heat release function coefficients from experimental data}

In order to solve the specific problem for the given method the calculation program for computer facilities, after which, varying the control actions and carrying out calculations of temperature field, the most efficient thermal treatment mode is selected.

After equation systems solving, according to the formulas (4-6) we have

$$
\frac{d Q}{d t}=Q_{\max } K\left(\frac{u-u_{f r}}{20-u_{f r}}\right)^{\zeta}\left(1-\frac{Q}{Q_{\max }}\right)^{v}
$$

Where:

- v-constant coefficient;

$-u_{f r}-$ concrete freezing point, ${ }^{\circ} \mathrm{C}$;

- $K, \zeta$ - coefficients that depend on the concrete mix composition.

Record the experimental data on the concrete heat release in the Table 1, where:

- $i$ - sequence number of measurement;

- $t_{i}$ - time point of $i$-th measurement from the beginning of hydration, h;

- $u_{i}$ - concrete temperature at the moment $\mathrm{t},{ }^{\circ} \mathrm{C}$;

- $Q_{i}$ - specific concrete heat release by the moment $\mathrm{t}, \mathrm{kJ} / \mathrm{m}^{3}$;

- $\left(\frac{d Q}{d t}\right)_{i}$ - concrete heat release intensity by the moment ti, $\mathrm{kW} / \mathrm{m}^{3}$. 
Table 1. Experimental data values on the concrete heat release.

\begin{tabular}{|c|c|c|c|c|}
\hline Number & $\boldsymbol{t}$ & $\boldsymbol{u}$ & $\boldsymbol{Q}$ & $\frac{d Q}{d t}$ \\
\hline 1 & $t_{l}$ & $u_{l}$ & $Q_{1}$ & $\left(\frac{d Q}{d t}\right)_{1}$ \\
\hline$i$ & $t_{i}$ & $u_{i}$ & $Q_{i}{ }^{*}$ & $\left(\frac{d Q}{d t}\right)_{i} *$ \\
\hline
\end{tabular}

Note that only $Q i$ or $\left(\frac{d Q}{d t}\right)_{i}$ can be measured in the experiment, then the other value is obtained by known recalculation.

To determine the coefficients $Q_{\max }, K, u_{f r}, \zeta, v$ it is recommended to use the following mathematical treatment method of experimental data. Using the heat release kinetics equation, experimental the function is drawn up:

$$
\sigma\left(Q_{\max }, K, u_{f r}, \zeta, v\right)=\sum_{i=1}^{N}\left[\left(\frac{d Q}{d t}\right)_{i}-Q_{\max } K\left(\frac{u-u_{f r}}{20-u_{f r}}\right)^{\zeta}\left(1-\frac{Q}{Q_{\max }}\right)^{v}\right]^{2}
$$

Function arguments (7) are the maximum specific heat release $Q_{\max }$ and heat release function coefficients. Minimum of function $\sigma$ is achieved at the desired values of these coefficients.

There are a number of searching methods for the minimum non-linear functions of several variables. Libraries of standard programs usually contain a program implementing one of these methods. For the independent preparation of such program, the most simple method of descent by coordinates can be recommended.

The above calculation method of heat release function coefficients provides a function that approximates in the best way the experimental dependence data of the heat release rate $\left(\frac{d Q}{d t}\right)_{i}$ from temperature $(u)$ and concrete heat release $(Q)$, with the minimum data available. It is enough to have the results of only one experiment on the concrete heat release in random temperature conditions covering the entire temperature range of interest. This is achieved by use of the recommended mathematic treatment method of experimental results.

\section{Conclusion}

Thus, the dynamics of temperature field is investigated using the example of special case with the preset variables that determine the concrete thermal treatment mode. It is evident that the boundary conditions and input data are always set again, based on the tasks provided for the solution that in turn indicates the applicability of proposed method, under different concrete thermal treatment modes.

During calculations with the use of software the limitation at the formulas set through a functional approach was observed, including the preset parameters passed to the subroutines as well as the need for duplication of variables that significantly complicated the set of program in Fortran language. This indicates the possibility of facilitating of the writing the program by selecting another software language, such as $\mathrm{C}++$. However, it 
should be noted that the calculating speed will not change much depending on the different software compilers selection [10].

As one can see, the numerical solution of model equations which allows calculating the temperature field in the hardening concrete structure by using computer software directly indicate the applicability of model in the development area of the most efficient modes without the need for volume calculations, which can significantly reduce the scientific experiment time.

Theoretical and practical significance of study results is the possibility to select the most efficient mode of thermal treatment of concrete structures, saving time for calculations, and, therefore, high quality of concrete with minimum thermal treatment duration and maximum reduction of energy costs is provided. Directly affecting the construction and installation works schedule and improving the construction quality of buildings and structures of reinforced concrete, basic requirements of energy efficiency and environmental friendliness of residential buildings in the implementation of the government program of the affordable housing market development are met [11].

\section{References}

1. S.O. Novikov, A.A. Sapukhin, Proc. of MSUCE 7, 96 (2013)

2. S.O. Novikov, Matec Web of Conf. 86, 04032 (2016)

3. N.S. Blokhina, AMM 405-408, 2687 (2013)

4. V. Garousi, M.V. Mäntylä, CSR 19, 56-77 (2016)

5. P. Élesztos, Proc. Of PPSME 48, 179-184 (2004)

6. V.S. Semenov, E.V. Tkach, S.A. Tkach, T.A. Rozovskaya, Proc. Eng. 111, 763 (2015)

7. B. Mahantaac, T.N. Singha, P.G. Ranjithb, EG 210, 104 (2016)

8. B.V. Zhadanovsky, S.A. Sinenko, AMR 838-841, 280 (2013)

9. J.J. Kim, K.-S. Youm, M.M. Taha, SWJ 2014, 9 (2014)

10. A.A. Volkov, A.V. Sedov, P.D. Chelyshkov, A.V. Doroshenko, AMM 580-583, 32313233 (2014)

11. B.V. Zhadanovsky, S.A. Sinenko, Science Review 9-2, 435 (2014) 